\title{
Calibrating the correlation between black hole mass and X-ray variability amplitude: X-ray only black hole mass estimates for active galactic nuclei and ultra-luminous $\mathrm{X}$-ray sources
}

\author{
Xin-Lin Zhou \\ Key Laboratory of Optical Astronomy, National Astronomical Observatories, Chinese \\ Academy of Sciences, Beijing 100012, China \\ Department of Physics and Tsinghua Center for Astrophysics, Tsinghua University, Beijing \\ 100084, China \\ Shuang-Nan Zhang \\ Key Laboratory of Particle Astrophysics, Institute of High Energy Physics, Chinese \\ Academy of Sciences, Beijing 100049, China \\ Physics Department, University of Alabama in Huntsville, Huntsville, AL 35899, USA \\ Ding-Xiong Wang \\ School of Physics, Huazhong University of Science and Technology, Wuhan 430074, China \\ Ling Zhu \\ Department of Physics and Tsinghua Center for Astrophysics, Tsinghua University, Beijing \\ 100084, China \\ zhouxl@nao.cas.cn
}

Received ; accepted 


\begin{abstract}
A calibration is made for the correlation between the X-ray Variability Amplitude (XVA) and black hole (BH) mass. The correlation for 21 reverberationmapped Active Galactic Nuclei (AGNs) appears very tight, with an intrinsic dispersion of 0.20 dex. The intrinsic dispersion of 0.27 dex can be obtained if $\mathrm{BH}$ masses are estimated from the stellar velocity dispersions. We further test the uncertainties of mass estimates from XVAs for objects which have been observed multiple times with good enough data quality. The results show that the XVAs derived from multiple observations change by a factor of 3 . This means that $\mathrm{BH}$ mass uncertainty from a single observation is slightly worse than either reverberation-mapping or stellar velocity dispersion measurements; however $\mathrm{BH}$ mass estimates with X-ray data only can be more accurate if the mean XVA value from more observations is used. With this calibrated relation, the $\mathrm{BH}$ mass and accretion rate may be determined for a large sample of AGNs with the planned International X-ray Observatory mission.

Proper interpretation of the first AGN X-ray quasi-periodic oscillation (QPO), seen in the Seyfert galaxy RE J1034+396, depends on its BH mass, which is not currently known very well. Applying this relation, the BH mass of RE J1034+396 is found to be $4_{-2}^{+3} \times 10^{6} M_{\odot}$. The high end of the mass range follows the relationship between the $2 f_{0}$ frequencies of high-frequency QPO and the BH masses derived from the Galactic X-ray binaries.

We also calculate the high-frequency constant $C=2.37 M_{\odot} \mathrm{Hz}^{-1}$ from 21 reverberation-mapped AGNs. As suggested by Gierliński et al., $M_{\mathrm{BH}}=C / C_{\mathrm{M}}$, where $C_{\mathrm{M}}$ is the high-frequency variability derived from XVA. Given the similar shape of power-law dominated X-ray spectra in ultra-luminous X-ray sources (ULXs) and AGNs, this can be applied to BH mass estimates of ULXs. We
\end{abstract}


discuss the observed QPO frequencies and BH mass estimates in the ULXs M82 X-1 and NGC 5408 X-1 and favor ULXs as intermediate mass BH systems.

Subject headings: accretion, accretion discs - galaxies: individual: RE J1034+396 $\mathrm{X}$-rays: galaxies $-\mathrm{X}$-rays: binaries 


\section{Introduction}

Active galactic nuclei (AGNs) are scaled-up versions of the Galactic X-ray binaries (XRBs), as recently evidenced by the physical link between the characteristic timescales and black hole (BH) masses corrected by the accretion rates (McHardy et al. 2006). The characteristic timescales are denoted by the break frequencies in the power spectral densities (PSDs). This suggests a similar broken power-law shape of PSDs from XRBs to AGN (e.g., Edelson \& Nandra 1999; Uttley, McHardy \& Papadakis 2002; Markowitz et al. 2003). The power spectra of XRBs also show multiple types of quasi-periodic oscillations (QPOs) other than the broadband noise. The first AGN QPO discovered in the Seyfert galaxy RE J1034+396 (Gierliński et al. 2008a) stresses the similarities between the XRBs and AGNs, but its proper interpretation is hammered by its unknown BH mass. RE J1034+396 is an extreme Narrow-Line Seyfert 1 galaxy (NLS1), with the BH mass remaining largely uncertain (e.g., Bian \& Zhao 2004). The BH mass determination is then a key issue since there are many controversies on the NLS1 mass estimates (e.g., Komossa \& Xu 2007; Marconi et al. 2008; Zhu et al. 2009).

We estimate the $\mathrm{BH}$ mass of $\mathrm{RE} \mathrm{J1034+396} \mathrm{from} \mathrm{the} \mathrm{X-ray} \mathrm{variability} \mathrm{amplitude}$ (XVA), $\sigma_{\text {rms }}^{2}$ (Nandra et al. 1997; Turner et al. 1999). A major difficulty in estimating BH masses from their XVA is the large scattering in the $M_{\mathrm{BH}}-\sigma_{\text {rms }}^{2}$ relation $(\mathrm{Lu} \& \mathrm{Yu} 2001$; Bian \& Zhao 2003; Papadakis 2004; O’Neill et al. 2005; Zhou et al. 2007; Miniutti et al. 2009; Nikolajuk et al. 2009). Note that the previous works were based on the BH masses derived from heterogeneous methods. This may cause additional scattering in the relation, since different methods of BH mass estimates may have quite different systematic errors. We therefore attempt to make a calibration of $M_{\mathrm{BH}}-\sigma_{\mathrm{rms}}^{2}$ correlation based on only the reverberation-mapped $\mathrm{BH}$ masses; we further check this calibration result with $\mathrm{BH}$ masses determined from the dispersion velocity measures of these AGNs' host galaxies. We also 
derive the high frequency constant as defined by Gierliński et al. (2008b). This is useful for the $\mathrm{BH}$ mass estimates of ultra-luminous X-ray sources (ULXs) in nearby galaxies, since both ULXs and AGNs have characteristic power-law dominated X-ray spectra.

This work is organized as follows. In Section 2, we re-analysis the $X M M$ Newton observations of RE J1034+396 and extract the light curves. In Section 3, we make a calibration of the correlation between the BH masses and XVAs and derive the high frequency constant. In Section 4, we discuss the uncertainties of mass estimates from XVA. In Section 5 we estimate BH mass for RE J1034+396 and discuss the implication of the AGN QPO. We find RE J1034+396 follows the relationship between the high-frequency QPO frequencies and BH masses derived from XRBs. Throughout this work, we use the cosmological parameters of $h_{0}=70 \mathrm{~km} \mathrm{~s}^{-1} \mathrm{Mpc}^{-1}, \Omega_{m}=0.27$, and $\Omega_{\Lambda}=0.73$.

\section{XMM-Newton observations of RE $\mathbf{J} 1034+396$}

XMM-Newton observed RE J1034+396 on 2002 May 01 for 15.7 ks and on 2007 May 31 for $93 \mathrm{ks}$. We screen the $93 \mathrm{ks}$ data with the SAS v7.0 software. The X-ray events corresponding to patterns $0-4$ for the EPIC PN data are selected. We extract the source light curves from a $45^{\prime \prime}$ circle of the detected source position for PN, with the background being taken from six source-free regions with the same size. The presence of background flares and data gaps in the final $7 \mathrm{ks}$ are checked and removed.

Figure 1 shows the extracted $0.3-10 \mathrm{keV}$ PN light curve $\left(128 \mathrm{~s} \mathrm{bin}^{-1}\right)$, which is similar in shape to that in Gierliński et al. (2008a). The light curve shows a periodic oscillation since $t_{0}=25 \mathrm{ks}$, with the frequency $f=1 / 3730 \mathrm{~s} \approx 2.7 \times 10^{-4} \mathrm{~Hz}$. Gierliński et al. (2008a) have adopted the method proposed by Vaughan (2005) to quantify the statistical significance of periodicities against an underlying continuum which involves 
dividing the periodogram by the best-fitting power law and using the known distribution of the periodogram ordinates to estimate the likelihood of observing a given peak. This method can be used to test the significance of candidate periodicities superposed on a red noise spectrum which has a power-law shape. The result indicates that the QPO is at $\sim 5.6$ $\sigma$ significance level.

\section{Calibrating $M_{\mathrm{BH}}-\sigma_{\mathrm{rms}}^{2}$ relation}

XVA is the variance of a light curve normalized by its mean squared after correcting for experimental noise. For a light curve segment with $N$ bins (Nandra et al. 1997; Turner et al. 1999),

$$
\sigma_{\mathrm{rms}}^{2}=\frac{1}{N \mu^{2}} \sum_{i=1}^{N}\left[\left(X_{i}-\mu\right)^{2}-\sigma_{i}^{2}\right],
$$

where $X_{i}$ and $\sigma_{i}$ are the counting rates and uncertainties in each bin. $\mu$ is the arithmetic mean of the counting rates.

Following O'Neill et al. (2005), the errors of $\sigma_{\text {rms }}^{2}$, which depend on the measurement uncertainties and the stochastic nature of the source, can be expressed as,

$$
\Delta_{\mathrm{tot}}\left(\sigma_{\mathrm{rms}}^{2}\right)=\sqrt{\left(\frac{\sigma_{\mathrm{frac}} \sigma_{\mathrm{rms}}^{2}}{\sqrt{N_{\mathrm{seg}}}}\right)^{2}+\left[\Delta_{\mathrm{boot}}\left(\sigma_{\mathrm{rms}}^{2}\right)\right]^{2}}
$$

where $N_{\text {seg }}$ is the number of available light-curve segments, $\sigma_{\text {frac }}$ is a fractional standard deviation, $\sigma_{\text {frac }}=0.74$ for $\log M_{\mathrm{BH}}>6.54$ and $\sigma_{\text {frac }}=0.48$ for $\log M_{\mathrm{BH}}<6.54 . \Delta_{\text {boot }}\left(\sigma_{\text {rms }}^{2}\right)$ is the bootstrap uncertainty which comes from the bootstrap simulation accounting for the measurement uncertainties.

XVA is calculated from a self-consistent analysis of an AGN's X-ray observation with a duration of at least $40 \mathrm{ks}$. The $2-10 \mathrm{keV}$ light curves are subdivided into segments with a duration of $39.936 \mathrm{ks}$ (binned at $256 \mathrm{~s}$ ). The mean XVA is used if there are multiple 
segments. We take the measured XVA listed in O'Neill et al. (2005) if available.

\subsection{Reverberation-mapped sample}

Homogeneous $\mathrm{BH}$ masses are required for studying and calibrating the $M_{\mathrm{BH}}-\sigma_{\mathrm{rms}}^{2}$ relation. BH masses based on the reverberation-mapping (RM) method for 35 AGN (Peterson et al. 2004) and the small Seyfert galaxy NGC 4395 (Peterson et al. 2005) are currently available. Here we use the reverberation-mapped BH masses for the calibration of the $M_{\mathrm{BH}}-\sigma_{\mathrm{rms}}^{2}$ relation. We list in Table 1 the 21 reverberation-mapped AGN with at least one segment of X-ray data longer than $40 \mathrm{ks}$.

In Figure 2, we show the correlation between XVA and the RM BH masses. We assume that there is a linear correlation between the $M_{\mathrm{BH}}$ and XVA, $y=\alpha+\beta x$, where $y=\log \left(M_{\mathrm{BH}} / M_{\odot}\right), x=\log \left(\sigma_{\text {rms }}^{2}\right)$, with the measurement errors of $\epsilon_{x_{i}}$ for $x_{i}$ and $\epsilon_{y_{i}}$ for $y_{i}$. It was shown that the Nukers' estimate is an unbiased slope estimator for the linear regression (Tremaine et al. 2002). The Nukers' estimate is based on minimizing,

$$
\chi^{2} \equiv \sum_{i=1}^{N} \frac{\left(y_{i}-\alpha-\beta x_{i}\right)^{2}}{\epsilon_{y i}^{2}+\beta^{2} \epsilon_{x i}^{2}} .
$$

We apply the Nukers' estimate to derive the parameter of $\alpha=4.85 \pm 0.20$ and $\beta=-1.05 \pm 0.08$ for the 21 RM AGNs. The minimum $\chi^{2}$ per degree of freedom is 1.6 , indicating that either the uncertainties in the $\mathrm{BH}$ masses are underestimated or there is an intrinsic dispersion for $\mathrm{BH}$ mass estimates.

To account for the intrinsic dispersion in the $M_{\mathrm{BH}}-\sigma_{\mathrm{rms}}^{2}$ relation, we replace $\epsilon_{y i}$ by $\left(\epsilon_{y i}^{2}+\epsilon_{0}^{2}\right)^{1 / 2}$, where $\epsilon_{0}$ represents the intrinsic dispersion; $\epsilon_{0}$ is adjusted so that the value of $\chi^{2}$ per degree of freedom is unity. This procedure is preferable if the individual error estimates $\epsilon_{y_{i}}$ are reliable (Tremaine et al. 2002). Adding an intrinsic dispersion of 0.18 
dex decreases the value of $\chi^{2}$ per degree of freedom to unity, and gives the best-fit results, $\alpha=4.97 \pm 0.26$, and $\beta=-1.00 \pm 0.10$. Then,

$$
M_{\mathrm{BH}}=10^{4.97 \pm 0.26}\left(\sigma_{\mathrm{rms}}^{2}\right)^{-1.00 \pm 0.10} M_{\odot} .
$$

Note that the errors of the RM BH mass for IC4329A and NGC 4593 are quite large (Peterson et al. 2004). We exclude these two objects to ensure reliable error estimates of BH masses. The intrinsic dispersion of the $M_{\mathrm{BH}}-\sigma_{\mathrm{rms}}^{2}$ relation is 0.20 dex for the rest of 19 AGNs. We also plot the objects with the BH masses estimated from the empirical virial relation; these objects (open circles in Figure 2) generally follow the best-fit correlation well.

\subsection{Sample with stellar velocity dispersions}

The key result we obtained is that the intrinsic dispersion of the $M_{\mathrm{BH}}-\sigma_{\mathrm{rms}}^{2}$ relation is quite small, no larger than the uncertainties of RM BH masses of $0.3-0.4$ dex (Peterson et al. 2004). We further check this result by using the BH masses estimated from the stellar velocity dispersions, since AGN hold the same relation between the BH masses and the stellar velocity dispersions (hereafter $M_{\mathrm{BH}}-\sigma_{*}$ relation) as the normal galaxies (Gebhardt et al. 2000; Ferrarese et al. 2001). In Table 2, we list 21 AGNs with the stellar velocity dispersion measurements; only four objects are different from that listed in Table 1.

Figure 3 shows the relation of XVA against the $\mathrm{BH}$ mass. The $\mathrm{BH}$ mass is estimated

from the relation $M_{\mathrm{BH}}=1.349 \times 10^{8}\left(\sigma_{*} / 200\right)^{4.02} M_{\odot}$ (Tremaine et al. 2002). However, there is evidence for large scattering at the low BH mass end in the $M_{\mathrm{BH}}-\sigma_{*}$ relation (Greene \& Ho 2006). We therefore use the RM mass of NGC 4395 (Peterson et al. 2005), and estimate the $M_{\mathrm{BH}}$ of MCG-6-30-15 from the empirical virial relation using the $\mathrm{H} \beta$ line (Zhou \& Wang 
2005). It is difficult to determine the actual errors of mass estimates in Table 2. Tremaine et al. (2002) claimed that the stellar velocity dispersion shows a Gaussian measurement error of 0.06 dex. Assuming no intrinsic dispersion in the $M_{\mathrm{BH}}-\sigma_{*}$ relation, the error of mass estimates from the stellar velocity dispersion should be $4.02 \times 0.06 \approx 0.24$ dex. Therefore, we add the error of \pm 0.24 dex for the BH masses in Table 2 .

We then apply Nukers' estimate to derive the parameter of $\alpha=4.91 \pm 0.24$ and $\beta=-1.04 \pm 0.09$. The minimum $\chi^{2}$ per degree of freedom is 1.8 , indicating that either the uncertainties in $M_{\mathrm{BH}}$ are underestimated or there is an intrinsic dispersion for $M_{\mathrm{BH}}$ estimates. We add an intrinsic dispersion of 0.27 dex to decrease the value of $\chi^{2}$ per degree of freedom to unity, and give the best-fit results, $\alpha=5.15 \pm 0.29$, and $\beta=-1.01 \pm 0.11$. Then,

$$
M_{\mathrm{BH}}=10^{5.15 \pm 0.29}\left(\sigma_{\mathrm{rms}}^{2}\right)^{-1.01 \pm 0.11} M_{\odot} .
$$

The results are similar to that from the RM sample. This is a natural result since the RM method has been calibrated in agreement with the $M_{\mathrm{BH}} \sigma_{*}$ relation (Peterson et al. 2004). If the errors of $M_{\mathrm{BH}}$ used here are underestimated, the intrinsic dispersion of the

$M_{\mathrm{BH}}-\sigma_{\mathrm{rms}}^{2}$ relation should be smaller. Since it is difficult to determine the actual errors of $M_{\mathrm{BH}}$ estimates used here, we tend to use the calibrated relation with the RM sample, i.e., Equation (4).

\subsection{Weak dependence on Eddington ratio}

$\sigma_{\text {rms }}^{2}$ may also depend on the second variable, Eddington ratio, $\dot{m}_{\mathrm{E}}$ (e.g., Papadakis 2004). Following McHardy et al. (2006), we hypothesize that $\log M_{\mathrm{BH}}=A+B \log \sigma_{\mathrm{rms}}^{2}+$ $C \log L_{\text {bol }}$. The fit is good with $A=-(0.23 \pm 0.04), B=-(0.87 \pm 0.12)$, and $C=0.12 \pm 0.03$. 
Using $\dot{m}_{\mathrm{E}}=L_{\mathrm{bol}} / L_{\mathrm{Edd}}$, then $\sigma_{\mathrm{rms}}^{2} \propto \dot{m}_{\mathrm{E}}^{0.14} / M_{\mathrm{BH}}$, indicating that the $\mathrm{BH}$ accretion rates only weakly affect the mass estimates.

We further explore the dependence of $\sigma_{\text {rms }}^{2}$ on the X-ray luminosity (e.g., Barr \& Mushotzky 1986; Liu \& Zhang 2008), FWHM of the broad components of the optical H $\beta$ lines (e.g., Leighly 1999), and the X-ray photon index (e.g., O’Neill et al. 2005). Results of partial correlation analysis show that the correlation between $\sigma_{\mathrm{rms}}^{2}$ and $M_{\mathrm{BH}}$ are the main correlation after removing the effects of other dependence (Table 3).

\subsection{Amplitude of High-frequency Variability}

Gierliński et al. (2008b) have argued that the XVA at high frequencies can be used as an estimator of a BH's mass. Their hypothesis is that there is a universal power spectral shape for BHs at high frequencies (e.g., Lawrence \& Papadakis 1993; Hayashida et al. 1998) above the PSD break frequency, $f_{b}$, as illustrated in Fig. 4. This universal form is roughly a power law with an index $\alpha=2$, then $P(f)=C_{M}\left(f / f_{r}\right)^{-2}$, where $f_{r}$ is an arbitrary frequency chosen to be $f_{r}=1 \mathrm{~Hz}$.

Then XVA is calculated between frequencies $f_{1}$ and $f_{2}$, with $f_{b} \leq f_{1}<f_{2}$,

$$
\sigma_{\mathrm{rms}}^{2}=\int_{f_{1}}^{f_{2}} P(f) d f=C_{M} f_{r}\left(\frac{f_{r}}{f_{1}}-\frac{f_{r}}{f_{2}}\right) .
$$

The assumption is that $C_{\mathrm{M}}$ is inversely proportional to the $\mathrm{BH}$ mass, $M_{\mathrm{BH}}=C / C_{\mathrm{M}}$, where $C$ is the high frequency constant. Gierliński et al. (2008b) calculated the $C$ values from the Galactic XRB Cyg X-1. Different $C$ values can be obtained if the data corresponding to the different spectral state of Cyg X-1 are used; $C=1.24 M_{\odot} \mathrm{Hz}^{-1}$ for the low hard state and $C=2.77 M_{\odot} \mathrm{Hz}^{-1}$ for the soft state.

We calculate $C$ from the 21 AGNs with the RM masses. We find $C=2.37 M_{\odot} \mathrm{Hz}^{-1}$, 
close to the value for the soft state of $\mathrm{Cyg} \mathrm{X}-1$. This means that $C$ is not sensitive to $M_{\mathrm{BH}}$ since the BH mass crosses over 8 orders of magnitudes from XRBs to AGNs. This also agrees with the fact that the amplitude of PSDs only slightly changes from XRBs to AGNs (see Fig. 18 in McHardy et al. 2004). Thus,, $C$ can be reasonably assumed to be constant for AGN mass estimates.

We emphasize that the $C$ value from AGNs can be also applied for the $\mathrm{BH}$ mass estimates of ULXs in nearby galaxies, because the X-ray spectral properties of ULXs are similar to that of AGNs. Both ULXs and AGNs show power-law dominated X-ray spectra, together with a soft X-ray excess. Also, both ULXs and AGNs lack the disk-emission-dominated state as frequently observed in the Galactic XRBs.

\section{5. $f_{1}$ test and origin of intrinsic dispersion}

In Figure 5 we show the dependence of XVA on $f_{1}$. If $f_{1} \ll f_{2}, \sigma_{\text {rms }}^{2} \approx C_{M} f_{r}^{2} / f_{1}$, where $f_{1}$ corresponds to the lowest frequency of data sampled. That means, XVA is inversely proportional to $f_{1}$ used. We calculate XVAs from a segment of $40 \mathrm{ks} X M M$-Newton data with a different $f_{1}$. XVAs generally decrease with the increasing $f_{1}$, but only a few AGNs follow the slope of -1 . The measured deviation from $\sigma_{\mathrm{rms}}^{2} \propto 1 / f_{1}$ may be due to either a red-noise component in the light curve (Vaughan et al. 2003), or generally the assumption of $\alpha=2, f_{b} \leq f_{1} \ll f_{2}$ is not always right for the sample. It was also known that the PSD of NGC 4051 shows a break frequency of $8 \times 10^{-4} \mathrm{~Hz}$ (McHardy et al. 2004), which is higher than the $f_{1}$ used (for this work, $f_{1}=1 / 39.936 \mathrm{ks}=2.5 \times 10^{-5} \mathrm{~Hz}$ ). However, PSDs of most of AGNs in the sample are still unknown. These may contribute to the intrinsic dispersion of the $M_{\mathrm{BH}}-\sigma_{\text {rms }}^{2}$ relation. 


\section{Errors of $M_{\mathrm{BH}}$ estimates from XVA}

The intrinsic dispersion of the $M_{\mathrm{BH}}-\sigma_{\mathrm{rms}}^{2}$ relation derived above is at a level of $0.2-0.3$ dex, no larger than the uncertainties of $\mathrm{BH}$ masses used here. Can the mass estimates from XVA be more accurate than those estimates from the empirical virial relation or from the stellar velocity dispersion? In this section we discuss the errors of the mass estimates from XVA.

X-ray PSDs of AGN are not stationary on the timescales probed by typical XMMNewton or Chandra observations. The red-noise nature of X-ray variability of AGN has been established (e.g., Lawrence et al. 1987; McHardy \& Czerny 1987). For the red-noise process, XVA depends on the shape of PSD, and the time resolution and duration of the light curve. XVA may change from one part of the light curve to the next, even when the variability is produced by a constant PSD. Thus, different XVA values can be obtained if different data are used. Therefore, we attempt to estimate the uncertainties of mass estimates by looking at objects which have been observed multiple times with good enough data quality to make independent $M_{\mathrm{BH}}$ estimates.

Figure 6 shows the distribution of XVA for the NLS1 galaxy MCG-6-30-15 and 1H0707-495. MCG-6-30-15 was observed using XMM-Newton starting on 2000 July 11 and ending on 2001 August 4, with a total exposure time of 480 ks. 1H0707-495 was observed using XMM-Newton starting on 2000 October 21 and ending on 2008 February 6, with a total exposure time of $\sim 800 \mathrm{ks}$. We analyze all these data and filter the light curves to remove any background flares. We then subdivide the light curves into the $39.936 \mathrm{ks}$ segments binned at $256 \mathrm{~s}$. The XVA derived from each data segment shows the variability by a factor of $3(\sim 0.5 \mathrm{dex})$. This is roughly consistent with the error estimates by Equation (2).

Gierliński et al. (2008b) have analyzed RXTE Proportional Counter Array observations 
of seven Galactic XRBs. The high-frequency variability derived from XVA changes by a factor of $2-3$, even when the data corresponding to different spectral state are used. Therefore the error of mass estimates from a single data segment is slightly larger than the RM method of $0.3-0.4$ dex, despite of the small intrinsic dispersion of the $M_{\mathrm{BH}}-\sigma_{\mathrm{rms}}^{2}$ relation. However, assuming XVA randomly scatters around the true value for the PSD of the process (Vaughan et al. 2003), the mean XVA of many data segments may reduce the error and thus the mass estimates can be more accurate than with the RM or stellar velocity dispersion method.

We would also like to point out that the RM AGNs are a subset of BHs which are skewed toward low mass, high Eddington fraction of AGNs. These are probably the bulk of the AGNs for which reliable XVA can be computed with the present instrumentation. It would be not safe to attempt to apply this method to the objects like M31* or Sgr A* with the same calibration, since they both have extremely low accretion rates. The XVA can be a function of the photon energy. Thus the choice of spectral bandpass affects the XVA estimates. Nevertheless we do not address this issue in the current work since all the results presented here are based on the $2-10 \mathrm{keV}$ band. However, if an X-ray instrument with a softer bandpass were used, or if one wanted to apply this method to higher redshift AGNs where both time dilation and redshifting of the X-rays were relevant, it would be necessary to develop an understanding of what would change. This will be investigated in a future work. 


\section{BH mass and QPO frequency}

\section{1. $\quad$ BH Mass of RE J1034+396}

The X-ray variability of RE J1034+396 increases with the photon energy (Middleton et al. 2009). We extract the $2-10 \mathrm{keV}$ light curve for the XVA calculation. The mean XVA of the two segments of light curves of RE J1034+396 is found to be $0.0234 \pm 0.0081$, corresponding to a $\mathrm{BH}$ mass of $4_{-2}^{+3} \times 10^{6} M_{\odot}$ (See Figure 2$)$.

BH masses in AGN are usually determined from two methods: RM method and the $M_{\mathrm{BH}}-\sigma_{*}$ relation. We apply the empirical virial relation based on the RM method firstly and then compare the result from the stellar velocity dispersion, in order to estimate the $\mathrm{BH}$ mass in RE J1034+396. The gas in the broad line region (BLR) are thought to be virialized. The virial BH mass can then be calculated from

$$
M_{\mathrm{BH}}=R_{\mathrm{BLR}} f^{2} \mathrm{FWHM}_{\mathrm{H} \beta}^{2} / G,
$$

where $\mathrm{FWHM}_{\mathrm{H} \beta}$ is the FWHM of the whole $\mathrm{H} \beta$. For an isotropic velocity distribution, as generally assumed, $f=\sqrt{3} / 2 . \quad R_{\text {BLR }}$ is obtained from the RM experiment or the $L_{5100}-R_{\mathrm{BLR}}$ relation calibrated with the RM method. The BH mass in RE J1034+396 calculated from Equation (7) is $6.3 \times 10^{5} M_{\odot}$, when $\mathrm{FWHM}_{\mathrm{H} \beta}=701 \mathrm{~km} \mathrm{~s}^{-1}$ is used (Bian \& Zhao 2004). However, recently it has been found that for NLS1s, the broad H $\beta$ line can be usually decomposed with a double Gaussian model, i.e., a very broad gaussian component (VBGC) and an intermediate gaussian component (IMGC) (Mullaney \& Ward 2008; Zhu et al. 2009). It is suggested that the BH mass should be calculated with FWHM of the VBGC (Zhu et al. 2009). Then, the virial BH mass should be corrected as

$$
M_{\mathrm{BHb}}=R_{\mathrm{VBLR}} f^{2} \mathrm{FWHM}_{\mathrm{H} \beta}^{2}\left(\mathrm{FWHMb}_{\mathrm{FWHM}} \mathrm{FW}^{2} / G,\right.
$$

where $\mathrm{FWHMb}$ is $\mathrm{FWHM}_{\mathrm{H} \beta}$ of the $\mathrm{VBGC}$ and $R_{\mathrm{VBLR}}$ is the radius of the very broad line 
region (VBLR). $R_{\mathrm{BLR}}$, is used as $R_{\mathrm{VBLR}}$, because RM usually measures the size of the VBLR (Zhu et al. 2009). Its $\mathrm{H} \beta$ line can be well decomposed into three components as shown in Mason et al (1996): a narrow line component, VBGC and IMGC. Bian \& Huang (2009) have recently analyzed the SDSS spectrum of RE J1034+396, by properly modeling the spectrum of its host galaxy. They found that the FWHM of the VBGC is $1690 \pm 296$ $\mathrm{km} \mathrm{s}^{-1}$. We use Equation (8) to correct the BH mass, $M_{\mathrm{BHb}}=(1690 / 701)^{2} \times 6.3 \times 10^{5}$ $M_{\odot}=3.7 \times 10^{6} M_{\odot}$. Considering the possible intrinsic scattering in the virial mass determination, the mass range of RE J1034+396 should be $2-7 \times 10^{6} M_{\odot}$. This mass is in excellent agreement with the result from XVA, also roughly consistent with the range of $1-4 \times 10^{6} M_{\odot}$, determined from the stellar velocity dispersion of the host galaxy of RE J1034+396 (Bian \& Huang 2009).

\subsection{QPO in RE $\mathrm{J} 1034+396$}

Figure 7 shows the scale of accretion flows between the $\mathrm{BH}$ mass and the QPO frequency across six orders of magnitudes. For a typical high-frequency (HF) QPO, $f_{0}=931\left(M_{\mathrm{BH}} / M_{\odot}\right) \mathrm{Hz}$ (Remillard \& McClintock 2006), where $f_{0}$ is the fundamental frequency. This relation is derived from three Galactic XRBs, XTE J1550-564, GRO J1655-40 and GRS 1915+105, which display a pair of HFQPOs with 3:2 frequency ratios. The frequencies of XRBs are plotted for the stronger QPO that represent $2 \times f_{0}$. If the

QPO in RE J1034+396 is a typical high-frequency QPO with $f=2 f_{0}$ or $f=3 f_{0}$, it would correspond to a $\mathrm{BH}$ mass of $6.9 \times 10^{6} M_{\odot}$ or $1.0 \times 10^{7} M_{\odot}$, respectively. The high end of mass range of RE J1034+396 favors this AGN QPO as a HFQPO with $f=2 f_{0}$. Middleton \& Done (2009) suggested that the QPO is the AGN analog of the $67 \mathrm{~Hz}$ QPO in GRS $1915+105$, and not the HFQPOs seen in 3:2 ratios. However, that one scales the QPO frequency for the $\left(4 \times 10^{6} / 14\right)$ mass ratio between RE J1043+396 and GRS 1915+105, one 
gets $76 \mathrm{~Hz}$ - within $\sim 10 \%$ of $67 \mathrm{~Hz}$, and well within the error bars on the BH mass for RE $\mathrm{J} 1043+396$.

HFQPOs with the harmonic pairs of frequencies in a 3:2 ratio were often suggested to arise from some type of resonance mechanism (e.g., Abramowicz \& Kluzniak 2001; Wang et al. 2007), where the frequencies are correlated with the BH mass and spin. The BH spin only changes the resonance orbits of accreting matter by a factor of a few. These may be the underlined physical reasons for the precise scale of accretion flows between the HFQPO frequency and BH mass. Rezzolla et al. (2003) proposed a model which requires that the two frequencies being oscillation modes of the same torus, rather than requires a resonance between the two QPO frequencies. The advantage of the Rezzolla et al. model for explaining what is seen here is that the higher frequency QPO is not seen, and the strengths of the different modes can vary independently. An alternative explanation for not seeing the higher frequency QPO might be that the PSD has not been made in the $10-30$ $\mathrm{keV}$ range where that QPO is usually seen in the Galactic XRBs, so this is not conclusive evidence in favor of the Rezzolla model over the resonance models. The QPOs previously seen are stellar mass BHs, which form through stellar evolution and grow insubstantially. They might all have quite similar spins. AGN, which can grow by both mergers and accretion, could have a different range of $\mathrm{BH}$ spin. Hence even if the QPO is due to the same mechanism as what produces the 3:2 frequency ratio QPOs in XRBs, a very realistic possibility is that the $\mathrm{BH}$ in RE J1043+396 has a mass closer to the most likely mass of $4 \times 10^{6} M_{\odot}$ from the XVA analysis, but has a lower spin than the stellar mass BHs.

\subsection{QPOs in ULXs}

There are QPOs observed in the ULX M82 X-1 (Strohmayer \& Mushotzky 2003; Feng \& Kaaret 2007) and NGC 5408 X-1 (Strohmayer et al. 2007), but the BH masses of these 
two ULXs were estimated ranging from 25 to $10^{5} M_{\odot}$ (e.g., Dewangan et al. 2006; Wu \& Gu 2008, etc). The non-standard accretion disk scenario (e.g., Soria et al. 2007) can explain the low soft X-ray temperature of M82 X-1 without invoking the intermediate mass BH. However, by assuming the M82 X-1 similar to the low-luminosity hard state of Galactic XRBs, Yuan et al. (2007) derived a mass of $9-50 \times 10^{4} M_{\odot}$ for M82 X-1 by fitting the Chandra X-ray data together with the constraints of radio and infrared upper limits using the advection-dominated accretion flow model. Lower $\mathrm{BH}$ mass $\left(10^{4} M_{\odot}\right)$ can be obtained if M82 X-1 corresponds to the high-luminosity hard state. Assuming the QPOs in M82 X-1 and NGC 5408 X-1 are low-frequency QPOs, Casella et al. (2008) placed the constraints on the $\mathrm{BH}$ masses of $100-1300 M_{\odot}$. Obviously, other possible QPO identifications will yield to different mass estimates. From the correlation between the spectral slope and the QPO frequency (known to exist in Galactic XRBs, see e.g. Vignarca et al. 2003), Fiorito \& Titarchuk (2004) derived a mass of the order of $10^{3} M_{\odot}$ for M82 X-1, although no such correlation was found in ULXs. Recently, Strohmayer \& Mushotzky (2009) found that the X-ray spectral and timing properties of NGC 5408 X-1 are quite analogous to the Galactic XRBs in the "very high" or "SPL" state. They also derived the high-frequency variability, $C_{\mathrm{M}}$ (Gierliński et al. 2008b) for mass estimates. If we adopt the high-frequency constant $C=2.37 M_{\odot} \mathrm{Hz}^{-1}$ from this work, their $C_{\mathrm{M}}$ values correspond to mass ranges of $3200-4000 M_{\odot}$ and $8400-10800 M_{\odot}$ for the two observations. This is not far from the scale between the HFQPO frequencies and the BH masses.

\section{Conclusions}

Calibration of the correlation between the black hole mass and the XVA shows that this correlation is quite tight, with an intrinsic dispersion of around 0.2 dex. The intrinsic dispersion may be caused by the uncertain shape of power spectral densities and the 
stochastic nature of the red-noise light curves. The independent mass estimates from the X-ray variability derived from multiple observations show the uncertainties by a factor of 3 ( $\sim 0.5$ dex). This is slightly larger than the errors of reverberation mapping or the stellar velocity dispersion measurements. However, the BH mass estimates can be more accurate if using the mean XVA derived from multiple observations. $\mathrm{BH}$ accretion rates only weakly affect the mass estimates. We find the high frequency constant $C=2.37 M_{\odot} \mathrm{Hz}^{-1}$ from the reverberation-mapped AGN. Given the similar shape of power-law dominated X-ray spectra in Ultra-Luminous X-ray sources and AGN, this can be applied to mass estimates of ULXs in nearby galaxies. The BH mass of RE J1034+396 is found to be $4_{-2}^{+3} \times 10^{6} M_{\odot}$ from the XVA. The high end of mass range of RE J1034+396 follows the relation between the high-frequency QPO frequencies and the BH masses derived from the Galactic X-ray binaries, suggesting the precise scaling of accretion flows among them. We also discuss the observed QPO frequencies and BH mass estimates in the ULX M82 X-1 and NGC 5408 X-1 and favor the intermediate mass BH systems.

We also find that X-ray variability as the mass estimator is independent of the luminosity, the distance and the viewing angle of the source; where luminosity is calculated by assuming the isotropy of emission. This is not true in many cases. Jets and outflows are common features and thus the beaming effect may play a role. The viewing angle may also change the inferred luminosity (Zhang 2005) considerably, since the X-ray emission from an accretion disk is generally not isotropic. Nevertheless, XVA provides quite reliable BH mass measurements without knowing the "true" luminosity of the source. Conventionally the BH mass in an AGN is often estimated from the measures of its broad line's width which may depend on the viewing angle of the source, unless the broad line region is completely spherically symmetric. For example, a narrower line profile can be obtained if it is seen from the pole-on angle and the BLR is flattened with a substantial Keplerian motion component. The radiation pressure may also affect the scale of the BLRs in AGNs. Apparently, XVA is 
independent of these effects. Therefore, the method of estimating BH's masses with XVAs may be a good alternative for NLS1 galaxies and ULXs, whose BH mass estimates have remained largely uncertain so far. Long and deep X-ray survey observations may allow the $\mathrm{BH}$ masses and thus accretion rates determined for a large sample of AGN, with, for example, the planned International X-ray Observatory.

We are very grateful to an anonymous referee for many useful comments and suggestions, which allowed us to improve the manuscript substantially. We thank useful discussions with Bian, W.-H., Feng, H., Laor, A., Li, T. P., Liu, Y., Lu, Y., Soria, R., Wang, J.-M., Ye, Y.-C., Zhang, Y.-H. and Zhao, Y.-H. We also thank Tremaine, S. to answer our questions on the fitting algorithm. This work was supported, in part, by China postdoctoral science foundation, Directional Research Project of the CAS under project No. KJCX2-YW-T03, the National Natural Science Foundation of China under grant Nos. 10821061, 10733010, 10725313, and 973 Program of China under grant 2009CB824800. 


\section{REFERENCES}

Abramowicz, M. A., \& Kluźniak, W. 2001, 374, 19

Barr, P., \& Mushotzky, R. F. 1986, Nature, 320, 421

Bian, W., \& Zhao, Y. 2003, MNRAS, 343, 164

Bian, W., \& Zhao, Y. 2004, MNRAS, 352, 823

Bian, W., \& Huang, K. 2009, MNRAS, 401, 507

Casella, P., Ponti, G., Patruno, A., Belloni, T., Miniutti, G., \& Zampieri, L. 2008, MNRAS, 387,1707

Dewangan, G. C., Titarchuk, L., \& Griffiths, R. E. 2006, ApJ, 637, 21

Edelson, R., \& Nandra, K. 1999, ApJ, 514, 682

Feng, H., \& Kaaret, P. 2007, ApJ, 668, 941

Ferrarese, L., Pogge, R. W., Peterson, B. M., Merritt, D., Wandel, A., \& Joseph, C. L. 2001, ApJ, 555, L79

Fiorito R., \& Titarchuk L., 2004, ApJ, 584, L5

Gebhardt, K. et al. 2000, ApJ, 543, L5

Gierliński, M., Middleton, Ma., Ward, M., \& Done, C. 2008a, Nature, 455, 369

Gierliński, M., Nikolajuk, M., \& Czerny, B. 2008b, MNRAS, 383, 741

Greene, J. E., \& Ho, L. C. 2006, ApJ, 641, L21

Hayashida, K., Miyamoto, S., Kitamoto, S., Negoro, H., \& Inoue, H. 1998, ApJ, 500, 642 
Komossa, S., \& Xu, D. 2007, ApJ, 667, 33

Lawrence, A., Watson, M., Pounds, K., \& Elvis, M. 1987, Nature, 325, 694

Lawrence, A., \& Papadakis, I. 1993, ApJ, 414, L85

Leighly, K. M. 1999, ApJS, 125, 297

Liu, Y., \& Zhang, S. N. 2008, A\&A, 480, 699

Lu, Y. J., \& Yu, Q. J. 2001, MNRAS, 324, 653

Marconi, A., Axon, D. J., Maiolino, R., Nagao, T., Pastorini, G., Pietrini, P., Robinson, A., \& Torricelli, G. 2008, ApJ, 678, 693

Mason, K. O. et al. 1996, MNRAS, 283, L26

Markowitz, A. et al. 2003, ApJ, 593, 96

McHardy, I., \& Czerny, B. 1987, Nature, 325, 696

McHardy, I. M., Papadakis, I. E., Uttley, P., Page, M. J., \& Mason, K. O. 2004, MNRAS, 348,783

McHardy, I. M., Koerding, E., Knigge, C., Uttley, P., \& Fender, R. P., 2006, Nature, 444, 730

Middleton, M., Done, C., Ward, M., Gierliński, M., \& Schurch, N. 2009, MNRAS, 394, 250

Middleton, M., \& Done, C. 2009, MNRAS, preprint(astro-ph/0908.0224)

Miniutti, G., Ponti, G., Greene, J. E., Ho, L. C., Fabian, A. C., \& Iwasawa, K. 2009, MNRAS, 394, 443

Mullaney, J. R., \& Ward, M. J. 2008, MNRAS, 385, 53 
Nandra, K., George, I. M., Mushotzky, R. F., Turner, T. J., \& Yaqoob, T. 1997, ApJ, 476, 70

Nelson, C. H., \& Whittle, M. 1995, ApJS, 99, 67

Nikolajuk, M., Czerny, B., \& Gurynowicz, P. 2009, MNRAS, 394, 2141

Oliva, E., Origlia, L., Maiolino, R., \& Moorwood, A. F. M. 1999, A\&A, 350, 9 O

O'Neill, P. M., Nandra, K., Papadakis, I. E., \& Turner, T. J. 2005, MNRAS, 358, 1405

Papadakis, I. E. 2004, MNRAS, 348, 207

Peterson, B. M. et al. 2004, ApJ, 613, 682

Peterson, B. M. et al. 2005, ApJ, 632, 799

Remillard, R. A., \& McClintock, J. E. 2006, ARA\&A, 44, 49

Rezzolla, L., Yoshida, S., Maccarone, T. J., \& Zanotti, O. 2003, MNRAS, 344, L37

Soria, R., Goncalves, A. C., \& Kuncic, Z. 2007, AIPC, 924, 919

Strohmayer, T. E., \& Mushotzky, R. F. 2003, ApJ, 586, L61

Strohmayer, T. E., Mushotzky, R. F., Winter, L., Soria, R., Uttley, P., \& Cropper, M. 2007, ApJ, 660, 580

Strohmayer, T. E., \& Mushotzky, R. F. 2009, ApJ, 703, 1386

Tremaine, S. et al. 2002, ApJ, 574, 740

Turner, T. J., George, I. M., Nandra, K., \& Turcan, D. 1999, ApJ, 524, 667

Uttley, P., McHardy, I. M., \& Papadakis, I. E. 2002, MNRAS, 332, 231 
Vaughan, S., Edelson, R., Warwick, R. S., \& Uttley, P. 2003, MNRAS, 345, 1271

Vaughan, S. 2005, A\&A, 431, 391

Vignarca F., Migliari S., Belloni T., Psaltis D., \& van der Klis M., 2003, A\&A, 397, 729

Wang, D. X., Ye, Y. C., \& Huang, C. Y. 2007, ApJ, 657, 428

Wu, Q. W., \& Gu, M. F. 2008, ApJ, 682, 212

Yuan, F., Taam, R. E., Misra, R., Wu, X. B., \& Xue, Y. Q. 2007, ApJ, 658, 282

Zhang, S. N. 2005, ApJ, 618, L79

Zhou, X. L., \& Wang, J. M. 2005, ApJ, 618, L83

Zhou, X. L., Yang, F., Lv, X. R., \& Wang, J. M. 2007, AJ, 133, 432

Zhu, L., Zhang, S. N., \& Tang, S. M. 2009, ApJ, 700, 1173 


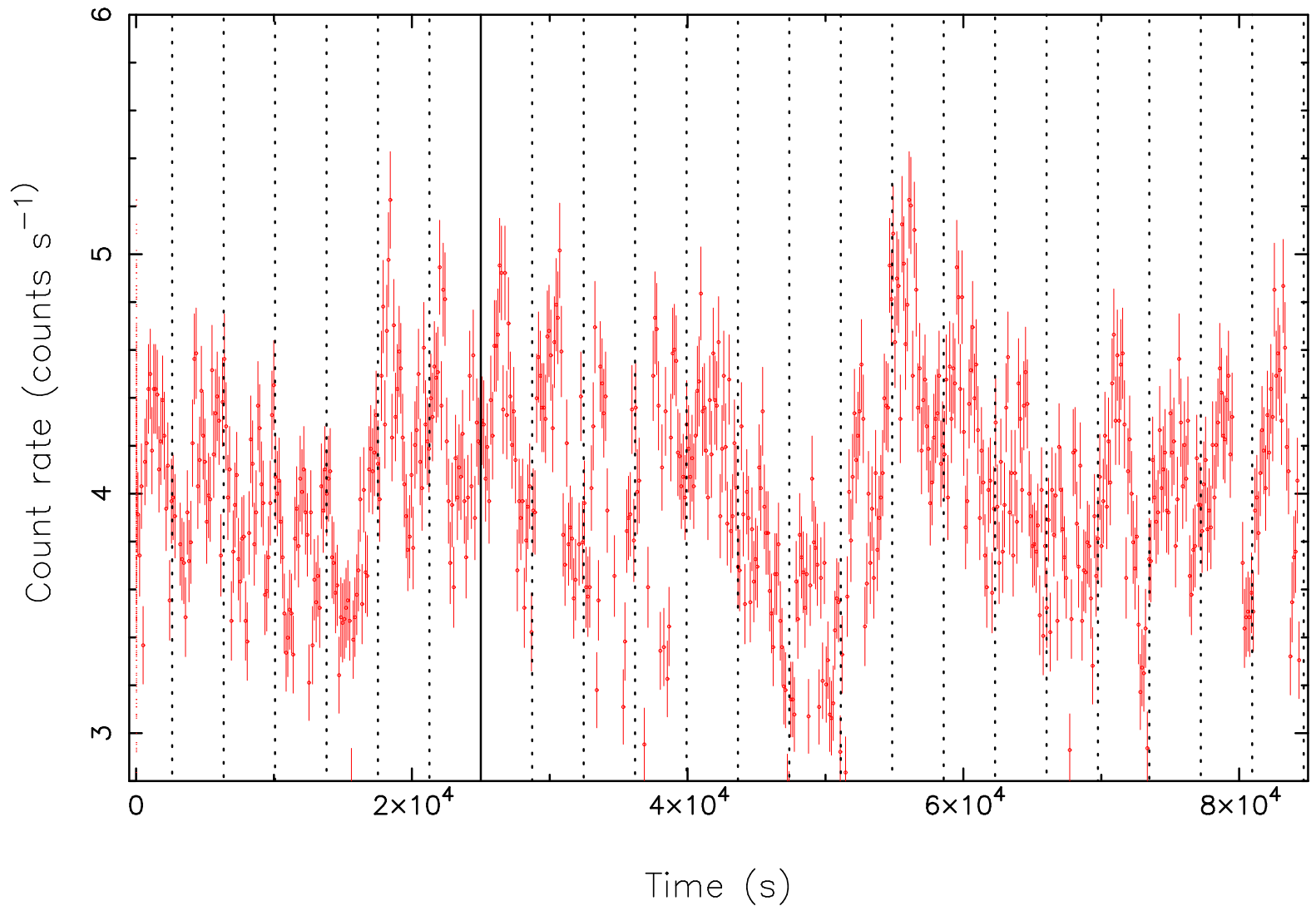

Fig. 1.- The source light curve extracted from the XMM-Newton PN camera in the $0.3-10$ $\mathrm{keV}$ band binned at $128 \mathrm{~s}$ for RE J1034+396. It is similar in shape to that in Gierliński et al. (2008a). It shows a periodic oscillation of $T=3730 \mathrm{~s}$ since $t_{0}=25 \mathrm{ks}$. 


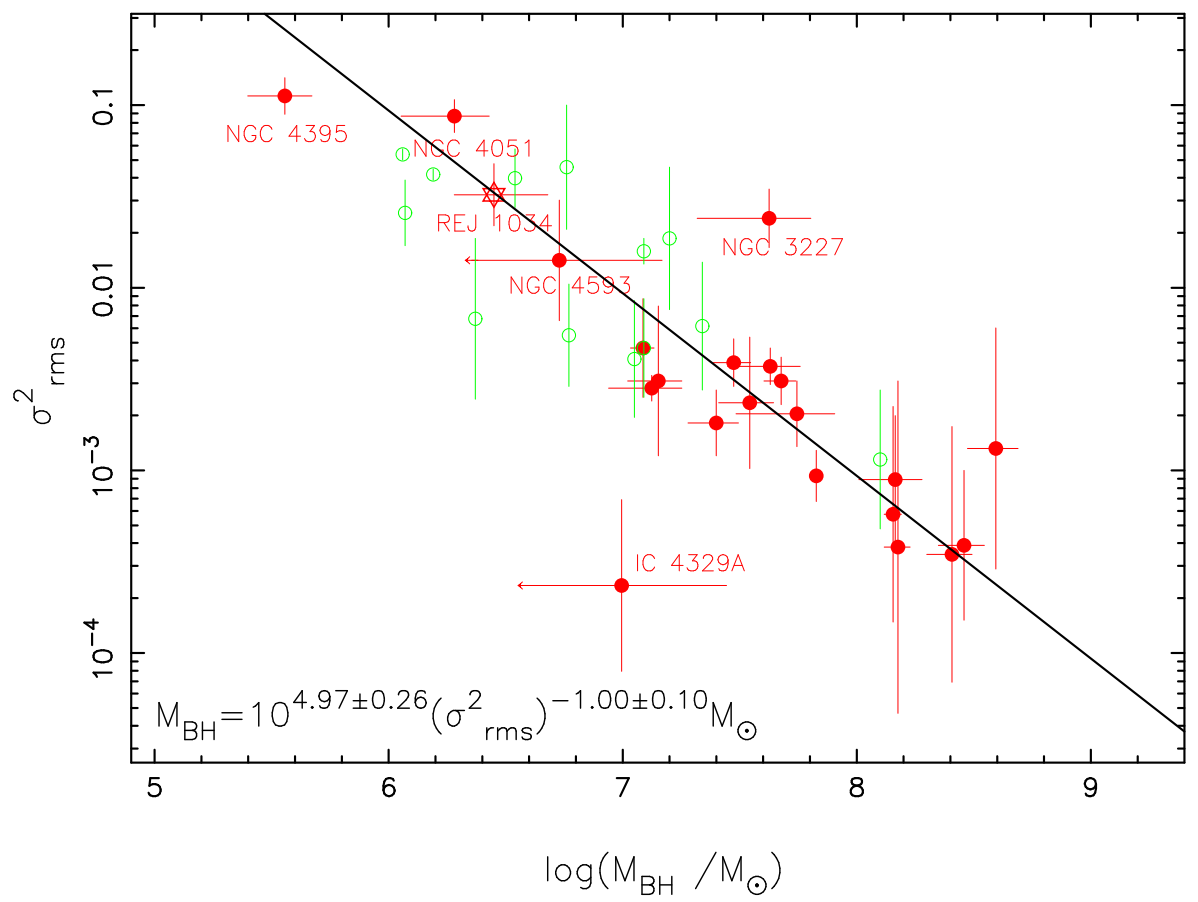

Fig. 2.- Correlation between the X-ray variability amplitude and the BH mass. The filled points denote the 21 objects with the $\mathrm{BH}$ masses measured from the reverberation-mapping method. The best-fit linear relation given by the Nukers' estimate for the 21 AGN is also shown in the plot. The intrinsic dispersion of this fit is 0.2 dex. Most of objects show negligible scattering compared with the linear fit. The star denotes RE J1034+396. We also plot the objects with the mass estimated from the empirical virial relation; these objects (open circles) generally well follow the linear fitting relation. 


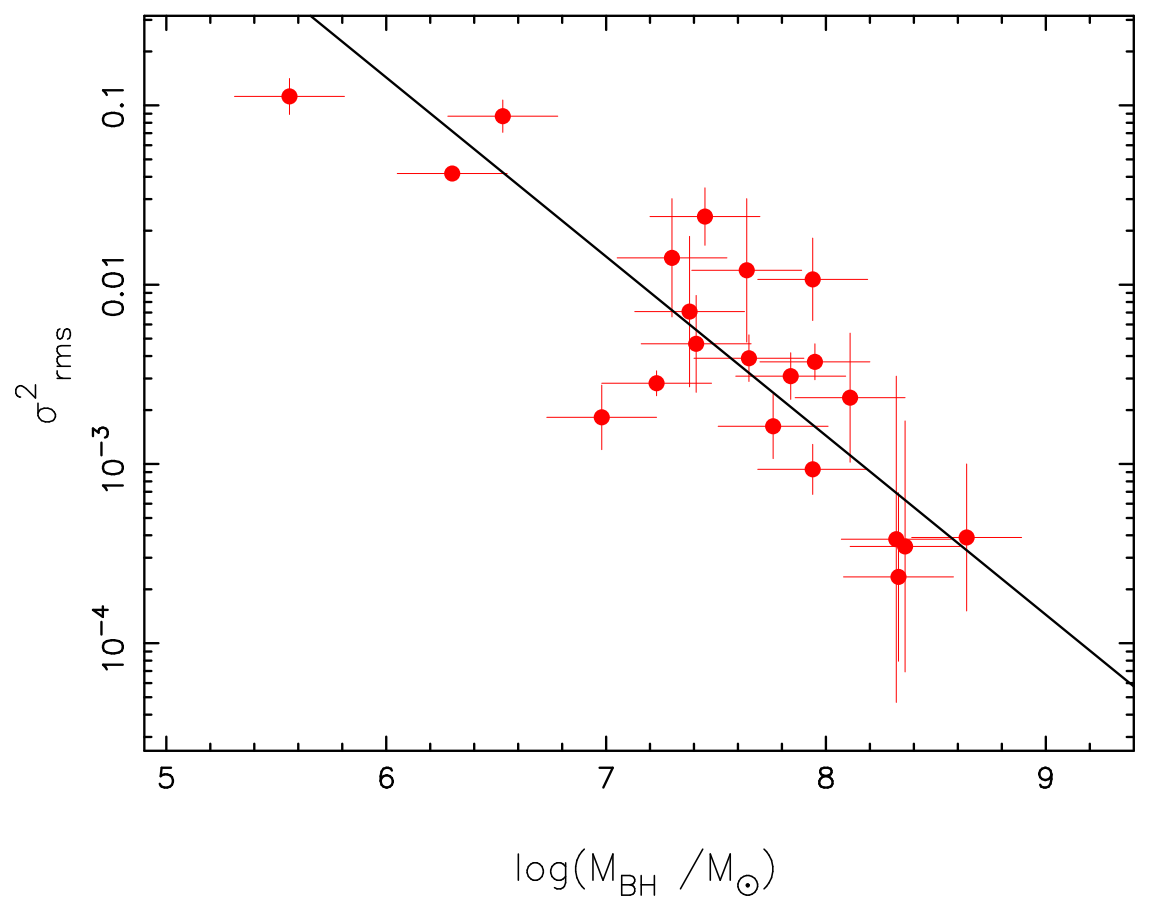

Fig. 3.- Correlation between the X-ray variability amplitude and the $\mathrm{BH}$ mass. The $\mathrm{BH}$ masses are mainly estimated from the stellar velocity dispersion measurements, with an assumed error of 0.24 dex. See the text for the details. The Nuker's estimates give the similar results to that in Fig. 2, with an intrinsic dispersion of 0.27 dex. 


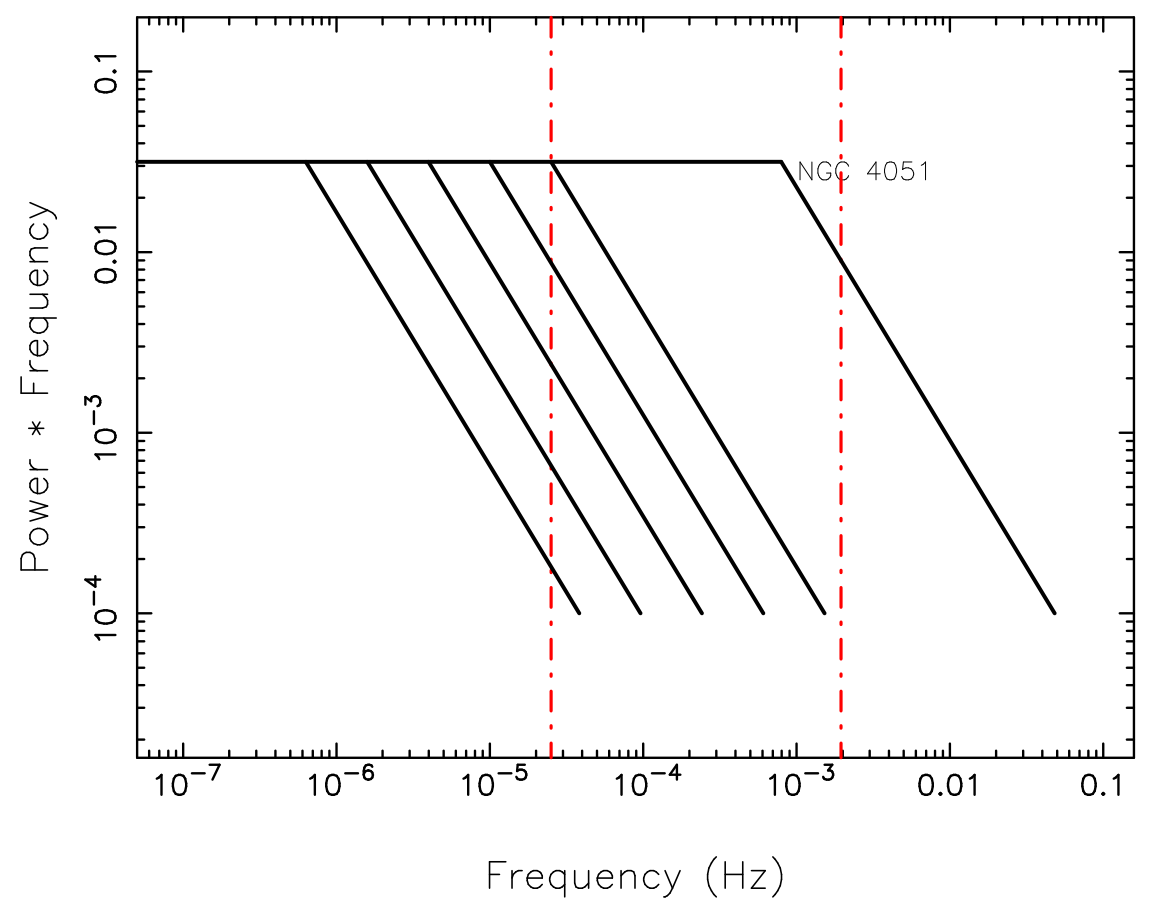

Fig. 4.- The assumed power-law shape of the power spectral densities (PSD) at high frequency. The two dot-dashed lines denote the low frequency $f_{1}$ and the high frequency $f_{2}$ sampled. For this work, $f_{1}=1 / 39.936 \mathrm{ks}=2.5 \times 10^{-5} \mathrm{~Hz}$, and $f_{2}=1 / 2 \times 256 \mathrm{~s}=2.0 \times 10^{-3}$ Hz. To ensure the estimation of the integral over the power law PSD, the frequency break should be lower than $f_{1}$. However, NGC 4051 has a higher frequency break of $8 \times 10^{-4} \mathrm{~Hz}$. This may cause the deviation of NGC 4051 in the $M_{\mathrm{BH}}-\sigma_{\mathrm{rms}}^{2}$ correlation. 


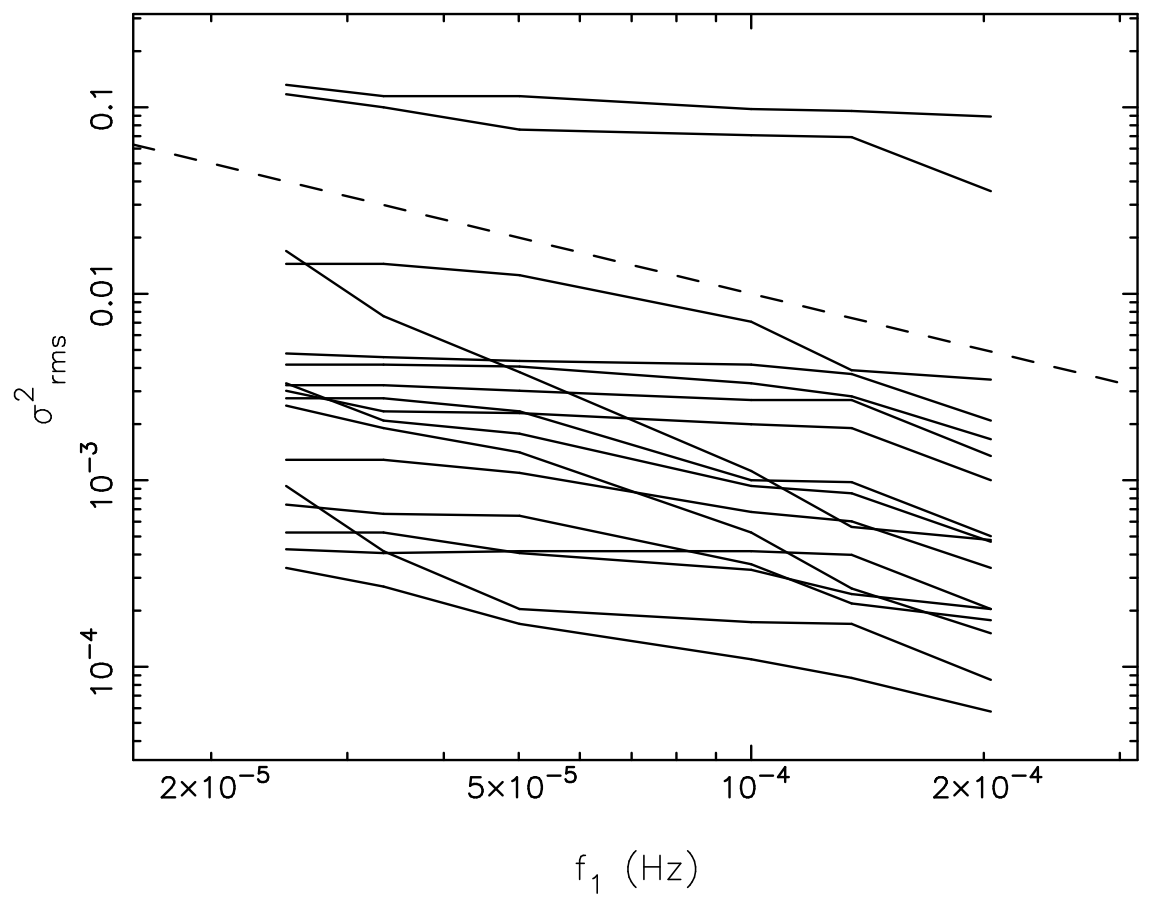

Fig. 5. - Dependence of X-ray variability amplitude on $f_{1}$ from a segment of $40 \mathrm{ks} X M M$ Newton data. $\sigma_{\text {rms }}^{2}$ generally decreases with the increasing $f_{1}$, but only a few AGN follow the slope of -1 (dashed line). 


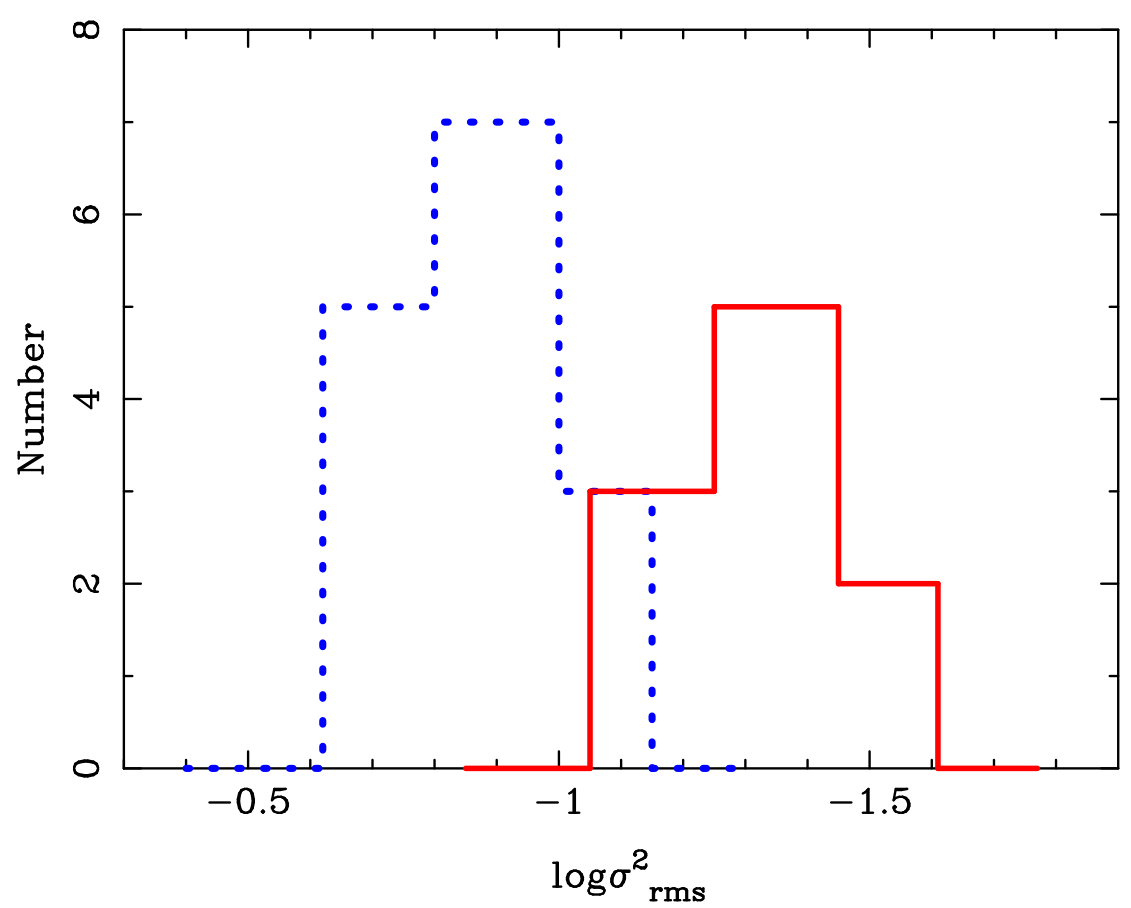

Fig. $\quad$ 6.- Distribution of X-ray variability amplitude derived from multiple $X M M$ Newton observations for the NLS1 galaxy MCG-6-30-15 (solid line) and 1H0707-495 (dotted line). 


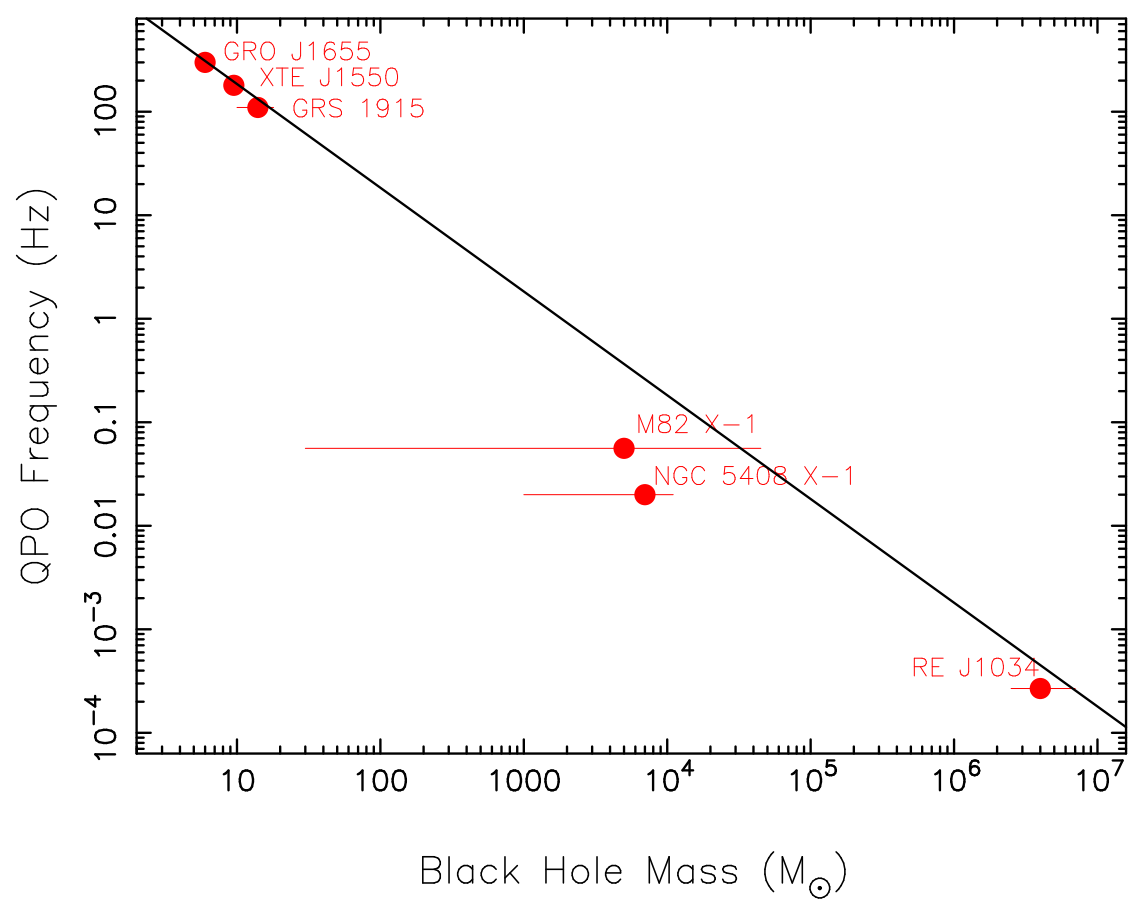

Fig. 7.- Relationship between the quasi-periodic oscillation (QPO) frequency and the BH mass. Three X-ray binaries, XTE J1550-564, GRO J1655-40 and GRS 1915+105 display a pair of high-frequency QPOs with a 3:2 frequency ratio. The frequencies of X-ray binaries are plotted for the stronger $\mathrm{QPO}$ that represent $2 \times f_{0}$. The line denotes the relation, $f(H z)=1862\left(M_{\mathrm{BH}} / M_{\odot}\right)^{-1}$ (Remillard \& McClintock 2006). The observed QPO frequency in the ULX M82 X-1 and NGC 5408 X-1 is also plotted. See the text for details. 
Table 1. Sample of AGN with Reverberation-Based mass

\begin{tabular}{|c|c|c|c|c|c|c|}
\hline $\begin{array}{l}\text { Name } \\
(1)\end{array}$ & $\begin{array}{l}z \\
(2)\end{array}$ & $\begin{array}{l}M_{\mathrm{BH}} \\
(3)\end{array}$ & $\begin{array}{l}\log \sigma_{\mathrm{rms}}^{2} \\
(4)\end{array}$ & $\begin{array}{c}\text { Num. Seg. } \\
\text { (5) }\end{array}$ & $\begin{array}{r}\log L_{\mathrm{bol}} \\
(6)\end{array}$ & $\begin{array}{c}\log \dot{m}_{\mathrm{E}} \\
(7)\end{array}$ \\
\hline Mrk 335 & 0.02578 & $14.2 \pm 3.7$ & $-2.51 \pm 0.41$ & 1 & 44.81 & -0.44 \\
\hline PG $0026+129$ & 0.14200 & $393 \pm 96$ & $-2.88 \pm 0.66$ & 3 & 45.97 & -0.72 \\
\hline Fairall 9 & 0.04702 & $255 \pm 56$ & $-3.46 \pm 0.70$ & 6 & 45.20 & -1.31 \\
\hline Mrk 590 & 0.02638 & $47.5 \pm 7.4$ & $-2.51 \pm 0.13$ & 2 & 44.76 & -1.02 \\
\hline $3 \mathrm{C} 120$ & 0.03301 & $55.5_{-22.5}^{+31.4}$ & $-2.79 \pm 0.18$ & 4 & 45.12 & -0.72 \\
\hline Ark 120 & 0.03230 & $150 \pm 19$ & $-3.42 \pm 0.91$ & 2 & 45.18 & -1.10 \\
\hline Mrk 110 & 0.03529 & $25.1 \pm 6.1$ & $-2.74 \pm 0.18$ & 1 & 44.67 & -0.83 \\
\hline NGC 3227 & 0.00386 & $42.2 \pm 21.4$ & $-1.62 \pm 0.16$ & 4 & 43.33 & -2.40 \\
\hline NGC 3516 & 0.00884 & $42.7 \pm 14.6$ & $-2.43 \pm 0.10$ & 18 & 43.83 & -1.89 \\
\hline NGC 3783 & 0.00973 & $29.8 \pm 5.4$ & $-2.41 \pm 0.13$ & 8 & 44.21 & -1.36 \\
\hline NGC 4051 & 0.00234 & $1.91 \pm 0.78$ & $-1.06 \pm 0.09$ & 6 & 42.88 & -1.50 \\
\hline NGC 4151 & 0.00332 & $13.3 \pm 4.6$ & $-2.55 \pm 0.07$ & 29 & 43.83 & -1.39 \\
\hline PG $1211+143$ & 0.08090 & $146 \pm 44$ & $-3.05 \pm 0.35$ & 3 & 45.70 & -0.56 \\
\hline NGC 4395 & 0.00106 & $0.36 \pm 0.11$ & $-0.95 \pm 0.10$ & 6 & 41.42 & -2.24 \\
\hline NGC 4593 & 0.00900 & $5.36_{-6.95}^{+9.37}$ & $-1.85 \pm 0.33$ & 1 & 44.03 & -0.79 \\
\hline IC $4329 \mathrm{~A}$ & 0.01605 & $9.90_{-11.88}^{+17.88}$ & $-3.63 \pm 0.47$ & 6 & 44.27 & -0.83 \\
\hline Mrk 279 & 0.03045 & $34.9 \pm 9.2$ & $-2.63 \pm 0.36$ & 1 & 44.83 & -0.81 \\
\hline NGC 5548 & 0.01718 & $49.4 \pm 7.7$ & $-3.03 \pm 0.14$ & 16 & 44.46 & -1.47 \\
\hline 3C 390.3 & 0.05610 & $287 \pm 64$ & $-3.41 \pm 0.41$ & 2 & 44.90 & -1.66 \\
\hline Mrk 509 & 0.03440 & $143 \pm 12$ & $-3.24 \pm 0.59$ & 2 & 45.23 & -1.03 \\
\hline NGC 7469 & 0.01632 & $12.2 \pm 1.4$ & $-2.33 \pm 0.27$ & 2 & 44.67 & -0.49 \\
\hline
\end{tabular}

(1) Object name. (2) Redshift. (3) Black hole mass in units of $10^{6} M_{\odot}$, taken from Peterson et al. (2004) and Peterson et al. (2005). (4) Log of the mean X-ray variability amplitude. (5) Number of usable light-curve segments (39.936 ks per segment). (6) Log of bolometric luminosity. (7) Log of the Eddington ratio, $\dot{m}_{\mathrm{E}}=L_{\mathrm{bol}} / L_{\mathrm{Edd}}$. 
TABle 2. SAmple of AGN With STEllar Velocity Dispersions

\begin{tabular}{|c|c|c|c|c|c|c|}
\hline $\begin{array}{l}\text { Name } \\
\text { (1) }\end{array}$ & $\begin{array}{l}z \\
(2)\end{array}$ & $\begin{array}{c}\log \left(M_{\mathrm{BH}} / M_{\odot}\right) \\
(3)\end{array}$ & $\begin{array}{l}\sigma_{*} \\
(4)\end{array}$ & $\begin{array}{l}\text { Ref. } \\
(5)\end{array}$ & $\begin{array}{l}\log \sigma_{\mathrm{rms}}^{2} \\
(6)\end{array}$ & $\begin{array}{c}\text { Num. Seg. } \\
\text { (7) }\end{array}$ \\
\hline Fairall 9 & 0.04702 & 8.36 & 228 & 4 & $-3.46 \pm 0.70$ & 6 \\
\hline Mrk 590 & 0.02638 & 7.84 & 169 & 2 & $-2.51 \pm 0.13$ & 2 \\
\hline Mrk 1040 & 0.01665 & 7.64 & 151 & 2 & $-1.92 \pm 0.40$ & 1 \\
\hline $3 \mathrm{C} 120$ & 0.03301 & 7.76 & 162 & 1 & $-2.79 \pm 0.18$ & 4 \\
\hline Ark 120 & 0.03230 & 8.32 & 224 & 1 & $-3.42 \pm 0.91$ & 2 \\
\hline Mrk 79 & 0.02219 & 7.38 & 130 & 3 & $-2.15 \pm 0.42$ & 1 \\
\hline Mrk 110 & 0.03529 & 6.99 & 87 & 1 & $-2.74 \pm 0.18$ & 1 \\
\hline NGC 3227 & 0.00386 & 7.45 & 135 & 1 & $-1.62 \pm 0.16$ & 4 \\
\hline NGC 3516 & 0.00884 & 7.95 & 181 & 1 & $-2.43 \pm 0.10$ & 18 \\
\hline NGC 3783 & 0.00973 & 7.65 & 152 & 4 & $-2.41 \pm 0.13$ & 8 \\
\hline NGC 4051 & 0.00234 & 6.53 & 80 & 3 & $-1.06 \pm 0.09$ & 6 \\
\hline NGC 4151 & 0.00332 & 7.23 & 119 & 2 & $-2.55 \pm 0.07$ & 29 \\
\hline NGC 4395 & 0.00106 & 5.56 & 30 & 1 & $-0.95 \pm 0.10$ & 6 \\
\hline NGC 4593 & 0.00900 & 7.30 & 124 & 2 & $-1.85 \pm 0.33$ & 1 \\
\hline MCG-6-30-15 & 0.00775 & 6.30 & 159 & 4 & $-1.38 \pm 0.03$ & 48 \\
\hline IC $4329 A$ & 0.01605 & 8.34 & 225 & 4 & $-3.63 \pm 0.47$ & 6 \\
\hline NGC 5506 & 0.00618 & 7.94 & 180 & 4 & $-1.97 \pm 0.23$ & 2 \\
\hline Mrk 279 & 0.03045 & 8.11 & 197 & 1 & $-2.63 \pm 0.36$ & 1 \\
\hline NGC 5548 & 0.01718 & 7.94 & 180 & 3 & $-3.03 \pm 0.14$ & 16 \\
\hline 3C 390.3 & 0.05610 & 8.64 & 268 & 1 & $-3.41 \pm 0.41$ & 2 \\
\hline NGC 7469 & 0.01632 & 7.41 & 133 & 1 & $-2.33 \pm 0.27$ & 2 \\
\hline
\end{tabular}

(1) Object name. (2) Redshift. (3) Black hole mass, mainly estimated from the stellar velocity dispersion. See the text for the details. (4) stellar velocity dispersion, in unit of $\mathrm{km} \mathrm{s}^{-1}$. (5) references for stellar velocity dispersions, 1. Greene \& Ho (2006); 2. Nelson \& Whittle (1995); 3. Ferrarese et al. (2001); 4. Oliva et al. (1999). (6) Log of the mean X-ray variability amplitude. (7) Number of usable light-curve segments (39.936 ks per segment). 
TABle 3. Partial CORRELATION ANALysis

\begin{tabular}{lcllll}
\hline \hline$L_{2-10 \mathrm{keV}}, M_{\mathrm{BH}}$ & $r_{M \sigma}$ & $r_{L \sigma}$ & $r_{M L}$ & $r_{M \sigma, L}$ & $r_{L \sigma, M}$ \\
& -0.85 & -0.69 & 0.73 & -0.70 & -0.19 \\
\hline $\mathrm{FWHMH} \beta, M_{\mathrm{BH}}$ & $r_{M \sigma}$ & $r_{F \sigma}$ & $r_{M F}$ & $r_{M \sigma, F}$ & $r_{F \sigma, M}$ \\
& -0.85 & -0.52 & 0.33 & -0.84 & -0.48 \\
\hline$\Gamma_{2-10 \mathrm{keV}}, M_{\mathrm{BH}}$ & $r_{M \sigma}$ & $r_{\Gamma \sigma}$ & $r_{M \Gamma}$ & $r_{M \sigma, \Gamma}$ & $r_{\Gamma \sigma, M}$ \\
& -0.85 & -0.20 & $\ldots$ & $\ldots$ & $\ldots$ \\
\hline
\end{tabular}

$r$ is the correlation coefficient. $M, L, F$ and $\Gamma$ denotes $M_{\mathrm{BH}}, L_{2-10 \mathrm{keV}}, \mathrm{FWHMH} \beta$ and $\Gamma_{2-10 \mathrm{keV}}$, respectively. 